J. Lake Sci. (湖泊科学), 2016, 28(5): 1103-1114

DOI 10. 18307/2016. 0521

(C) 2016 by Journal of Lake Sciences

\title{
青海湖西岸镭同位素的解吸和扩散特征
}

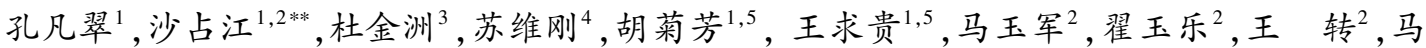 \\ 海英 ${ }^{6}$ \\ (1: 中国科学院青海盐湖研究所, 青海省盐湖地质与环境重点实验室, 西宁 810008) \\ (2: 青海师范大学, 青海省自然地理与环境过程重点实验室, 西宁 810008) \\ (3: 华东师范大学,河口海岸国家重点实验室,上海 200062) \\ (4: 青海省地震局预报中心,西宁 810001) \\ (5: 中国科学院大学, 北京 100049) \\ (6: 青海省德令哈市国土资源局,德令哈 817000)
}

摘 要: 对青海湖布哈河河口悬浮颗粒物、底部沉积物和青海湖湖底沉积物中的镭 (Ra) 同位素进行不同盐度和 $\mathrm{pH}$ 值的 解吸实验以及扩散实验,得到不同盐度湖水 $(2.8 \%, 5.8 \%, 8.8 \%$ 、 $11.8 \%$ 和 $14.8 \%$ ) 对悬浮颗粒物中镭的解吸活度, 和不同 时间段沉积物中镭同位素的扩散速率, 探讨盐度、 $\mathrm{pH}$ 值与颗粒物中镭同位素解吸的关系. 结果表明, ${ }^{224} \mathrm{Ra}$ 的解吸活度均 高于 ${ }^{226} \mathrm{Ra}$ 和 ${ }^{228} \mathrm{Ra}$ 的解吸活度; 在盐度为 $12 \%$ 附近时布哈河河口悬浮颗粒物中 ${ }^{223} \mathrm{Ra},{ }^{226} \mathrm{Ra}$ 和 ${ }^{228} \mathrm{Ra}$ 的解吸程度达到最大 值, 当盐度 $<9 \%$ 时, ${ }^{226} \mathrm{Ra}$ 解吸活度大于 ${ }^{228} \mathrm{Ra}$, 当盐度 $>9 \%$ 时, ${ }^{228} \mathrm{Ra}$ 的解吸活度大于 ${ }^{226} \mathrm{Ra}$, 这可能与当地岩石中富铀矿有 关. 河流沉积物 ${ }^{226} \mathrm{Ra}$ 和 ${ }^{228} \mathrm{Ra}$ 的扩散速率分别是 0.039 和 $0.290 \mathrm{dpm} /\left(\mathrm{m}^{2} \cdot \mathrm{h}\right)$; 湖底沉积物 ${ }^{226} \mathrm{Ra}$ 和 ${ }^{228} \mathrm{Ra}$ 的扩散速率分别 为 0.018 和 $0.092 \mathrm{dpm} /\left(\mathrm{m}^{2} \cdot \mathrm{h}\right)$, 湖底沉积物扩散速率小于河流沉积物扩散速率.

关键词: 镭同位素;解吸; 扩散;布哈河口; 青海湖

\section{Desorption and diffusion characteristics of radium isotopes from particles in the western part of Lake Qinghai}

KONG Fancui ${ }^{1}$, SHA Zhanjiang ${ }^{1,2 * *}$, DU Jinzhou ${ }^{3}$, SU Weigang ${ }^{4}$, HU Jufang ${ }^{1,5}$, WANG Qiugui ${ }^{1,5}$, MA Yujun $^{2}$, ZHAI Yule ${ }^{2}$, WANG Zhuan ${ }^{2} \&$ MA Haiying ${ }^{6}$

(1: Qinghai Institute of Salt Lakes, Chinese Academy of Sciences, Xining 810008, P.R.China)

(2: Key Laboratory of Natural Geography and Environmental Process in Qinghai Province Education, Qinghai Normal University, Xining 810008 , P.R.China)

(3: State Key Laboratory of Estuarine and Coastal Research, East China Normal University, Shanghai 200062, P.R.China)

(4: Qinghai Seismological Bureau Forecast Center, Xining 810001, P.R. China)

(5: University of Chinese Academy of Sciences, Beijing 100049, P.R.China)

(6: The Land and Resources Bureau of Delingha City, Delingha 817000 , P.R.China)

Abstract: Two laboratory experiments for Radium desorption and diffusion were conducted in this work, which included 1 ) the Radium desorption of sediments that were under different salinities $(2.8 \%$, 5.8\%o, 8.8\%o, $11.8 \%$ and $14.8 \%$ ) from the lake water and the potential of hydrogen from Buha River estuary. 2) Radium diffusive fluxes with time in the overlying water of lake sediments and in river sediments. We have got the desorbed activity of Radium from the suspended particles and Radium diffusion flu-

* 中国科学院百人计划项目“高原内陆盐湖水环境生物地球化学过程”、中国科学院青海盐湖研究所青年引导基金 B 类项目和青海省科技厅自然基金项目 “大柴旦周围水体中锂的地球化学分布规律及对盐湖补给通量研究” (2016-ZJ-921Q) 联合资助. 2015-08-21 收稿; 2015-11-16 收修改稿. 孔凡翠( 1984 ), 女, 博士, 研究实习员; E-mail: kfc@isl.ac.cn.

** 通信作者;E-mail: zhanjiang@ sina.com. 
xes from surface sediments. The results show that the desorption activities of ${ }^{224} \mathrm{Ra}$ are higher than those of ${ }^{226} \mathrm{Ra}$ and ${ }^{228} \mathrm{Ra}$, and the desorption degrees of ${ }^{224} \mathrm{Ra},{ }^{226} \mathrm{Ra}$ and ${ }^{228} \mathrm{Ra}$ have reached the maximum value when the salinity near $12 \%$. The desorption of ${ }^{226} \mathrm{Ra}$ activities are greater than that of ${ }^{228} \mathrm{Ra}$ when salinity $<9 \%$, however, when salinity $>9 \%$, the desorption activities of ${ }^{226} \mathrm{Ra}$ is less than that of ${ }^{228} \mathrm{Ra}$, which may be associated with the local rich uranium. The diffusion fluxes of ${ }^{226} \mathrm{Ra}$ and ${ }^{228} \mathrm{Ra}$ of sediments from Buha River are 0.039 and $0.290 \mathrm{dpm} /\left(\mathrm{m}^{2} \cdot \mathrm{h}\right)$, respectively, and they are 0.018 and $0.092 \mathrm{dpm} /\left(\mathrm{m}^{2} \cdot \mathrm{h}\right)$ from Lake Qinghai, respectively. The Radium diffusion fluxes of sediments in Lake Qinghai are less than that of the river.

Keywords: Radium isotopes; desorption; diffusion; Buha River estuary; Lake Qinghai

自然界中天然存在 4 种镭 $(\mathrm{Ra})$ 同位素: ${ }^{226} \mathrm{Ra}\left(\mathrm{T}_{1 / 2}=1600 \mathrm{a}\right) 、{ }^{228} \mathrm{Ra}\left(\mathrm{T}_{1 / 2}=5.75 \mathrm{a}\right) 、{ }^{224} \mathrm{Ra}\left(\mathrm{T}_{1 / 2}=3.66 \mathrm{~d}\right)$ 和 $^{223} \operatorname{Ra}\left(\mathrm{T}_{1 / 2}=11.4 \mathrm{~d}\right)$, 虽然它们的物理、化学性质相同, 但核性质却具有较大差异 ${ }^{[1-3]}$, 它们半衰期不同, 而 且来源也不相同, 因而在水环境中呈现不同的地球化学行为 ${ }^{[4]}$. 不同的半衰期使得它们研究范围十分宽泛, 在海洋研究中被广泛应用于量化水岩交换速率 ${ }^{[2]}$, 水体混合 ${ }^{[4,5-8]}$, 地下水排放通量 ${ }^{[9-14]}$, 营养物质 $\mathrm{PO}_{4}^{3-}$ 、 $\mathrm{SiO}_{4}^{-} 、 \mathrm{DON}$ 和 TDN 通过地下水的输送通量 ${ }^{[15-16]}$, 间隙水/上覆水交换速率 ${ }^{[17-18]}$, 与其他核素或参数的和还可 应用于沉积速率及年代学 ${ }^{[19-21]}$ 等. 镭同位素在淡水环境吸附在固体颗粒物表面, 当固体颗粒物遇到咸水时, 随着水体的盐度升高、离子强度增大, 钙、镁等碱土金属离子对颗粒物上镭的竞争吸附作用增强, 导致其上 吸附的 $\mathrm{Ra}$ 会发生解吸, 从而以溶解态 $\mathrm{Ra}^{2+}$ 的形式进人水体 ${ }^{[22-23]}$. 镭的这种吸附-解吸行为受到多种因素的 影响,如水体盐度 ${ }^{[5,18,24-26]} 、 \mathrm{pH}$ 值 ${ }^{[27]}$ 、温度 ${ }^{[12,27] \text { 、氧化还原电位 }}{ }^{[26-28]}$ 、沉积物粒径 ${ }^{[18,27,29]}$ 、沉积物矿物组 分 ${ }^{[30]}$ 、铁锰氧化物含量 ${ }^{[28,31-33]}$ 以及 alpha 粒子反冲作用等 ${ }^{[34-35]}$. 这些因素的作用机制各异, 对镭在沉积物上 解吸行为形成的影响也不尽相同. 但是在淡水与咸水混合的地带主要受水体盐度和 $\mathrm{pH}$ 值的影响.

悬浮颗粒物上镭同位素的解吸过程发生较快,在几秒到几小时的时间内就完成,通常在淡咸水交汇的 几分钟内就能达到解吸量的 30\% 50\% ${ }^{[5]}$. 辽河口水体中的 ${ }^{224} \mathrm{Ra}$ 和 ${ }^{223} \mathrm{Ra}$ 随盐度变化呈不保守现象, 研究认 为盐度为 $10 \%$ 左右的海区镭同位素高峰值可能是悬浮颗粒物解吸造成的 ${ }^{[36]}$. 水体 $\mathrm{pH}$ 值影响颗粒物的表面 净电荷量, 在低 $\mathrm{pH}$ 值的水体环境中, 二价镭离子形成表面络合物的能力较低, 进行解吸的能力较强 ${ }^{[37-38]}$. Gonneea 等研究认为, 在水体 $\mathrm{pH}$ 值从 8 降低至 5 时, 粗砂上的镭较易发生解吸 ${ }^{[26]}$. 在青海湖研究区域,布哈 河河水的盐度变化范围为 $0.2 \%$ $0.6 \%$, 青海湖湖水盐度变化范围为 $10.10 \%$ $14.71 \%$, 从河水到湖水盐度 变化比较大, 盐度可能是青海湖镭解吸的一个很重要的影响因素. 但是前人研究内容多为研究区盐度或者 $\mathrm{pH}$ 值对颗粒物解吸活度的影响, 或者是海底沉积物扩散镭的活度, 在一个研究区同时进行镭同位素解吸和 扩散实验的报道较少. 而且前人对于沉积物中镭的吸附-解吸行为的研究都是在沿海地区, 而在高原内陆咸 水湖的研究还未见报道.

本文为了更好地研究镭同位素的来源,在已知河流溶解态、地下水、湖泊表层水中镭同位素活度的基础 上, 拟通过开展不同盐度和 $\mathrm{pH}$ 值条件下, 青海湖布哈河河口悬浮颗粒物中雷同位素的解吸实验, 河流底部沉 积物以及湖底沉积物中镭同位素的扩散活度实验,推算高原内陆咸水湖泊周围河流悬浮颗粒物中镭同位素的 解吸与盐度、 $\mathrm{pH}$ 值之间的关系和扩散活度值特征, 通过对沉积物中镭的解吸量和扩散量进行研究, 从而为辨 别青海湖水体中镭同位素的来源、评估人湖水体停留时间、示踪沿岸地下水的排放通量推算研究提供科学依据.

\section{1 理论模型及实验过程}

\section{1 解吸实验的理论模型及过程}

在青海湖流域河流人湖处, 布哈河河水的盐度均小于 $1 \%$, 而青海湖的盐度为 $14 \%$ 左右, 在咸水和淡水 混合区, 在影响镭的吸附-解吸行为的众多因素中, 水体盐度的影响对镭的吸附-解吸行为最为重要 ${ }^{[17-18,26]}$, 我们就水体盐度梯度下镭在固-液两相的分配行为进行讨论, 以期为辨别镭同位素来源、示踪沿岸地下水排 放通量、评估水体交换时间以及估算沉积物-水界面交换通量等研究提供必要的定量依据.

Webster 等曾就水体盐度梯度下镭在颗粒物上的解吸行为, 建立了理论依据相对充分的数学模型, 并进 行室内解吸实验以验证模型的可靠性 ${ }^{[18]}$. 在该理论模型中, 认为受水体盐度变化的影响, 水体中镭的增加 可通过式 (1) 加以描述: 


$$
A_{\mathrm{w}}=\left[R+\frac{a}{b \cdot S}\right]^{-1} \cdot A_{0}
$$

其中, $S$ 为水体盐度值, 表示占标准海水的千分比, 单位为 $\% 0 ; A_{w}$ 为颗粒物解吸进人水体中镭的比活度, 单位 为 $\mathrm{dpm} / \mathrm{g} ; A_{0}$ 和 $a / b$ 为颗粒物的解吸参数, 两者数值大小取决于颗粒物的本身特性; $A_{0}$ 的物理意义为颗粒物 尚未发生解吸时, 单位面积的颗粒物上 “可交换态” 镭的活度量, 单位为 $\mathrm{dpm} / \mathrm{g} ; a / b$ 是公式推导过程中, 由 $\mathrm{Na}^{+}$和 $\mathrm{Ra}^{2+}$ 的吸附和解吸常数、颗粒物表面积、颗粒物表面能够吸附 $\mathrm{Na}^{+}$和 $\mathrm{Ra}^{2+}$ 最大个数等参数组成的函数, 无量纲 $; R$ 为解吸实验中水体体积与颗粒物体积的比值, 即固-夜比的倒数.

若根据式 (1) 指导解吸实验, 只需设定 $R$, 并测量已知盐度系列下的水体镭浓度 $A_{\mathrm{w}}$, 即可根据最小二乘 法确定颗粒物解吸参数 $a / b$ 和 $A_{0}$ 的数值, 此为第一种解吸实验方案, 通过不同盐度条件下颗粒物中镭的解 吸分析镭同位素在固-液两相的分配行为.

我们在 Webster 模型的基础之上进一步发展,同样可以计算颗粒物解吸参数 $a / b$ 和 $A_{0}$ 的数值. 其理论模 型具体如下: 颗粒物在某一特定盐度水体中第一次解吸, 其水体镭浓度上升至 $A_{w}^{1}$; 若将已解吸的悬浮颗粒物 在同一盐度水体中再次解吸, 水体镭浓度上升至 $A_{\mathrm{w}}^{2}$, 则水体镭浓度的增幅应以等比数列变化 ${ }^{[18]}$, 即:

$$
A_{\mathrm{w}}^{2}-A_{\mathrm{w}}^{1}=Q \cdot A_{\mathrm{w}}^{1}
$$

式中, $Q$ 为等比数列的公比数. 第 2 次沉积物解吸后水体镭浓度增幅需满足质量平衡, 即:

$$
\begin{aligned}
Q \cdot A_{\mathrm{w}}^{1}= & \left(R+\frac{a}{b \cdot S}\right)^{-1} \cdot A_{\mathrm{s}}^{1}=\left(R+\frac{a}{b \cdot S}\right)^{-1} \cdot A_{0}\left[1-R\left(R+\frac{a}{b \cdot S}\right)^{-1}\right]= \\
& A_{\mathrm{w}}^{1}\left[1-R\left(R+\frac{a}{b \cdot S}\right)^{-1}\right]
\end{aligned}
$$

由此确定：

$$
Q=1-R\left(R+\frac{a}{b \cdot S}\right)^{-1}
$$

根据递推法则, 第 $n$ 次解吸后水体镭浓度应等于该等比数列的前 $n$ 项之和, 即:

$$
A_{\mathrm{w}}^{n}=\frac{A_{\mathrm{w}}^{1}\left(1-Q^{n}\right)}{1-Q}=\frac{A_{0}}{R}\left(1-Q^{n}\right)
$$

根据以上理论推算, 颗粒物若在同一特定盐度的水体中多次解吸, 则根据每次解吸后水体镭浓度增加 趋势, 结合最小二乘法, 同样可以拟合出颗粒物解吸参数 $a / b$ 和 $A_{0}$ 的数值, 此为第 2 种解吸实验方案, 即用 同一个盐度对悬浮颗粒物进行多次淋洗分析镭同位素在固-液两相的分配行为.

本研究结合以上两种解吸实验方案思路, 即由第 2 种方案确定布哈河悬浮颗粒物中镭是否发生解吸, 然后由第一种方案确定镭同位素在固-液中的分配行为.

首先用青海湖湖水对布哈河河口悬浮颗粒物进行淋洗, 如果能从悬浮颗粒物上淋洗出镭同位素, 那说 明青海湖湖水会对河流悬浮颗粒物中的镭发生解吸, 否则将不会发生解吸. 淋洗实验过程: 选择盐度为 $14.8 \%$ 的青海湖湖水对悬浮颗粒物进行多次淋洗实验, 具体操作步骤如下:

1) 对采集的青海湖湖水用锰纤维进行过滤吸附其中含有的镭, 形成无镭水, 用圆柱形水桶取 $25 \mathrm{~L}$;

2) 将 $15 \mathrm{~g}$ 河流颗粒物置于水桶中搅拌 $2 \mathrm{~h}$, 静止沉淀 $1 \mathrm{~h}$;

3 ) 过滤上清液富集悬浮颗粒物解吸出来的镭;

4) 富集后立刻用 RaDeCC 器测试 ${ }^{224} \mathrm{Ra}$ 活度值;

5) 检测富集镭后的滤液盐度以确保未发生明显变化, 倒人水桶中再次摚拌 $2 \mathrm{~h}$ 进行淋洗, 如此反复 7 次, 每次淋洗后的滤液均采用同一根锰纤维柱富集, 并立即测量 ${ }^{224} \mathrm{Ra}$ 活度值, 直到镇纤维柱的 ${ }^{224} \mathrm{Ra}$ 活度不 再增加为止.

8 次淋洗实验在 $24 \mathrm{~h}$ 内连续快速完成, 并测试 ${ }^{224} \mathrm{Ra}$ 的活度, 以避免 ${ }^{224} \mathrm{Ra}$ 的衰减成为干扰因素.

随着不断对布哈河中悬浮颗粒物进行淋洗, 溶解态 ${ }^{224} \mathrm{Ra}$ 的活度不断增加, 但是当淋洗到第 7 次时, 溶解 态 ${ }^{22} \mathrm{Ra}$ 的活度呈现稳定趋势, 达到最大解吸活度 (3.592 dpm/g), 表明河流悬浮颗粒物中的镭被不断解吸到 河流水体中, 最后趋于稳定 (图 1). 


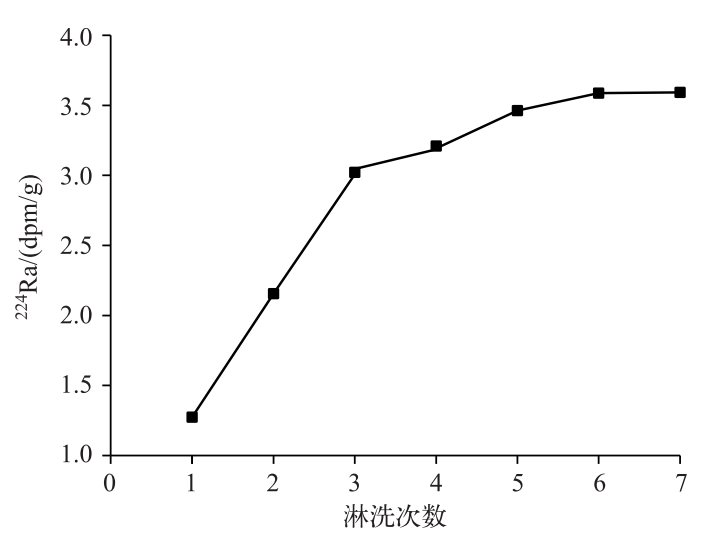

图 $1{ }^{224} \mathrm{Ra}$ 淋洗实验结果

Fig. 1 The result of ${ }^{224} \mathrm{Ra}$ leaching experiment

由淋洗实验可知, 河流悬浮颗粒物中的镭遇到 青海湖湖水会生解吸, 因此可以进行河流悬浮颗粒 物中镭的解吸实验, 不同盐度解吸操作过程为:

1) 配置无镭湖水. 经测定青海湖湖水盐度最大 值为 $14.8 \%$, 取足量盐度为 $14.8 \% 0$ 左右的湖水, 流过 装有 $\mathrm{MnO}_{2}$ 纤维柱子制成无镭湖水, 通过往无镭湖水 中加去离子水配置成盐度分别为 $11.8 \%$ 、 $8.8 \%$ 、 $5.8 \% 0 、 2.8 \%$ 、 $0.1 \%$ (盐度为 0 无法配制) 的无镭水， 每份体积为 $25 \mathrm{~L}$;

2) 取 6 份干的河流悬浮颗粒物, 每份 $10 \mathrm{~g}$ 置于 上述盐水中,利用搅拌机连续搅拌 $2 \mathrm{~h}$ 后静止沉淀, 搅拌强度以使颗粒物恰好悬浮为宜, 静止沉淀 $1 \mathrm{~h}$;

3) 用 $0.45 \mu \mathrm{m}$ 滤膜过滤上清液, 将收集的滤液 按照前面的方法用虹吸方式让沉积物上覆水缓慢流 过 $\mathrm{MnO}_{2}$ 纤维柱来富集镭, 立即测量 ${ }^{224} \mathrm{Ra}$ 活度值;

$4)$ 测完 ${ }^{224} \mathrm{Ra}$ 活度值的锰纤维进行共沉淀流程获得 $\mathrm{Ba}(\mathrm{Ra}) \mathrm{SO}_{4}$ 固体沉淀, 将沉淀放人 $\gamma$ 谱仪测试 ${ }^{226} \mathrm{Ra}$ 和 ${ }^{228} \mathrm{Ra}$ 的活度值.

\section{2 底部沉积物中镭同位素的扩散实验过程}

自然界存在的镭同位素由沉积物中母体 U-Th 衰变而来. 河口表层沉积物中 U-Th 衰变产生的镭, 通常 使得沉积物间隙水中镭活度大于河口水中的镭活度, 在活度差作用下, 间隙水中的镭扩散到河口水体中, 成 为河口水中镭的来源之一 ${ }^{[39]}$. 分别采集湖底沉积物和布哈河河口沉积物进行扩散培养实验. 把采集的表层 沉积物分别放置到直径为 25 和 $36 \mathrm{~cm}$ 的敞口塑料桶中, 厚度 $5 \sim 10 \mathrm{~cm}$, 对沉积物进行不同时段的扩散培养 实验. 为了使其充分稳定下来, 在实验之前将该样品放置 2 个月, 因为在取样和搬运过程中会对样品产生一 定扰动, 对测量结果有影响 ${ }^{[40]}$.

对湖底沉积物样品和河流沉积物样品分别进行 $0.5 、 1 、 2 、 4 、 7 、 12$ 和 $28 \mathrm{~d}$ 的扩散培养, 具体实验过程 为 ${ }^{[39]}$ : 在湖底沉积物扩散实验中: 沿着桶壁缓慢加入盐度为 $14.8 \%$ 的无镭湖水 $20 \mathrm{~L}$, 尽量不扰动沉积物, 放 置培养. 当达到培养时间后对水体中镭进行富集和测量. 之后, 再加人无镭湖水按照上述步骤进行下一个时 间段的扩散培养; 在河流沉积物扩散实验中: 每个时段开始时, 沿桶壁缓慢加人盐度为 $0.2 \%$ 的无镭河口水 $25 \mathrm{~L}$, 沉积物间隙水中的镭会逐渐扩散到该上覆水体中, 每个时段结束时, 对水体中的镭进行富集并测量其 活度, 然后再加人新的无镭河口水进行下一个时段的扩散培养, 在每次加人无镭河口水和富集水体中的镭 时, 都尽量减少对沉积物的扰动.

\section{2 样品采集及测试方法}

布哈河是青海湖湖区最大的地表径流, 位于青海湖西北部, 河长 $280 \mathrm{~km}$, 集流面积 $14300 \mathrm{~km}^{2}$, 约占青海 湖流域面积的一半, 水系呈不对称的羽状分布, 年平均径流量 $7.85 \times 10^{8} \mathrm{~m}^{3}$, 年总输沙量为 $3.55 \times 10^{5} \mathrm{t}$, 占总量 的 70\% 以上 ${ }^{[41]}$. 青海湖湖底沉积物扩散实验的沉积物是 2012 年 7 月在站位 $\mathrm{QH} 1 、 \mathrm{QH} 2 、 \mathrm{QH} 3$ (图 2) 用沉积 物采样器采集的 3 个沉积物柱子, 共 $2 \mathrm{~kg}$. 2014 年 8 月在布哈河选择河水盐度为 $0.1 \%$ (未找到盐度为 0 的 河水端元) 的站位 R1 过滤河水采集河流悬浮颗粒物 $5 \mathrm{~kg}$, 在站位 R2 和 R3(图 2) 采集河口区河流底部沉积 物共 $10 \mathrm{~kg}$, 分别用于河流悬浮颗粒物的解吸和河流底部沉积物的扩散实验, 其中站位 R2、R3 河水的盐度分 别为 $0.2 \% 、 0.3 \% 0, \mathrm{pH}$ 值分别为 $8.19 、 8.59$.

所有样品均通过锰纤维富集, 短半衰期 ${ }^{223} \mathrm{Ra}$ 和 ${ }^{224} \mathrm{Ra}$ 采用同步延时计数器 ( RaDeCC) 测量, 该仪器为美 国 Scientific Computer Instruments 公司生产的四通道同步延时计数器. 长半衰期 ${ }^{226} \mathrm{Ra}$ 和 ${ }^{228} \mathrm{Ra}$ 放射性活度的 测试用的是美国 ORTEC 公司生产的高纯锗 $\gamma$ 谱仪, 探测器为 GMX45P4 型, 所有实验和测试均在中国科学 院青海盐湖研究所完成. 


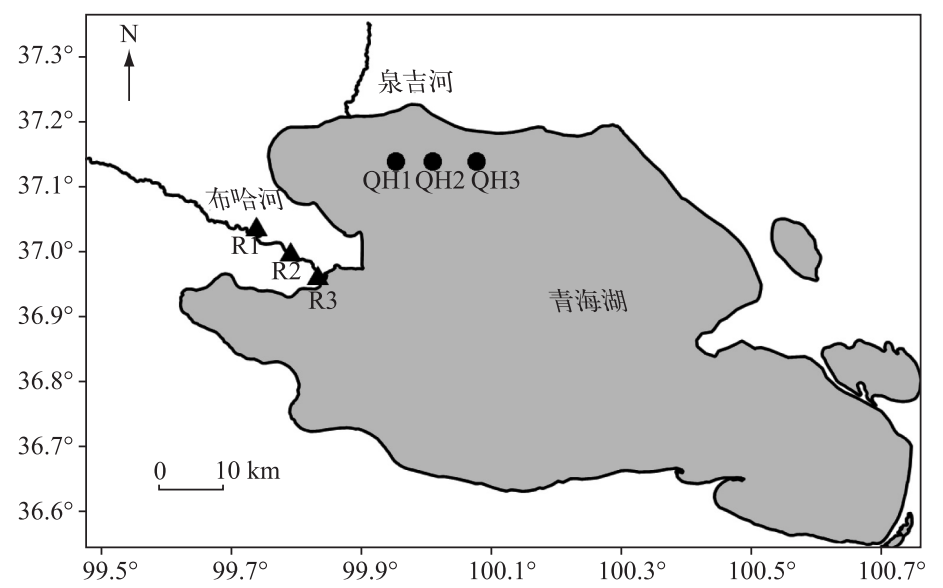

图 2 布哈河河口悬浮颗粒物、底部沉积物和青海湖湖底沉积物采样点位置

Fig. 2 Location of sampling sites of suspended particles and sediments of Buha River estuary and sediments of Lake Qinghai

\section{3 结果与讨论}

\section{1 悬浮颗粒物中镭同位素的解吸特征}

盐度是影响镭解吸的重要因素, 在一般封闭体系中, 沉积物中镭与其母体针处于平衡状态, 当遇到水体 盐度发生变化时, 如河流搬运悬浮颗粒物至河口盐度梯度区, 或海水倒灌人侵海岸含水层, 形成地下河口盐 度梯度区, 沉积物上的镭就会与海水中常量阳离子如 $\mathrm{Na}^{+}$等发生离子交换而进人到水体, 并随之迁移, 形成 沉积物中镭相对于母体钍亏损的现象 ${ }^{[18]}$. 布哈河河口悬浮颗粒物不同盐度 $(0 、 5.8 \%$ 、 $8.8 \%$ 、 $11.8 \% 0$ 和 $14.8 \%$ ) 所对应的悬浮颗粒物中 ${ }^{224} \mathrm{Ra}$ 的解吸活度分别为 $0.43 、 0.57 、 0.59 、 0.37$ 和 $0.32 \mathrm{dpm} / \mathrm{g}$, 盐度为 $2.8 \%$ 时 没有检测到 ${ }^{224} \mathrm{Ra}$ 的活度; 盐度为 $8.8 \%$ 、 $11.8 \%$ 和 $14.8 \%$ 所对应的 ${ }^{223} \mathrm{Ra}$ 的解吸活度分别为 $0.025 、 0.069$ 和 $0.036 \mathrm{dpm} / \mathrm{g}$, 盐度为 $2.8 \%$ 和 $5.8 \%$ 时没有检测到 ${ }^{223} \mathrm{Ra}$ 的活度; 各盐度所对应的 ${ }^{226} \mathrm{Ra}$ 的解吸活度分别为 $0.29 、 0.15 、 0.18 、 0.16 、 0.21$ 和 $0.13 \mathrm{dpm} / \mathrm{g}$; 各盐度所对应的 ${ }^{228} \mathrm{Ra}$ 的解吸活度分别为 $0.14 、 0.11 、 0.14 、 0.18 、 0.23$ 和 $0.17 \mathrm{dpm} / \mathrm{g}$. 布哈河悬浮颗粒物中 ${ }^{224} \mathrm{Ra}$ 的解吸活度均高于对应的 ${ }^{226} \mathrm{Ra}$ 和 ${ }^{228} \mathrm{Ra}$ 的解吸活度, 这可能与 ${ }^{224} \mathrm{Ra}$ 再生速率快有关 (表 1 ). 布哈河河口悬浮颗粒物中 ${ }^{226} \mathrm{Ra}$ 的解吸活度远小于海南东部八门湾悬浮颗粒

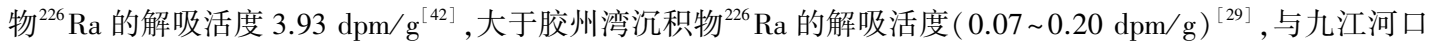
区悬浮颗粒物中 ${ }^{226} \mathrm{Ra}$ 的解吸活度 $(0.13 \sim 0.25 \mathrm{dpm} / \mathrm{g})$ 相接近 ${ }^{[39]}$ (表 1$)$. 说明本次实验获得的解吸数据是比 较可信的.

Gonneea 等发现当盐度升高时, 镭的解吸量具有增大的特点 ${ }^{[26]}$. 随着盐度的逐渐增加, Ra 同位素的解 吸活度先呈增加趋势, 说明盐度越高, 悬浮颗粒上解吸下来的 Ra 越多. 从理论上分析, 镭的解吸量不可能 无限制地增加, 随着盐度的继续增加, ${ }^{224} \mathrm{Ra}$ 的解吸活度表现为降低趋势 (图 3a). 当盐度 $<9 \%$ 时, ${ }^{226} \mathrm{Ra}$ 的解 吸活度大于 ${ }^{228} \mathrm{Ra}$, 当盐度 $>9 \%$ 时, ${ }^{228} \mathrm{Ra}$ 的解吸活度大于 ${ }^{226} \mathrm{Ra}$ (图 4). 在盐度为 $12 \%{ }^{\circ}$ 时, ${ }^{226} \mathrm{Ra}$ 和 ${ }^{228} \mathrm{Ra}$ 的解吸 活度达到了最大. 当盐度 $<4 \%$ 时, $R a$ 的解吸量增加较为缓慢, 当 $4 \% 0<$ 盐度 $<12 \%$ 时, Ra 的解吸量增加较迅 速, 可是当盐度 $>12 \%$ 时, Ra 的解吸量开始下降 (图 3a). 而其他地方的河流悬浮颗粒物 Ra 的解吸量随盐度 的变化有所不同, 在九江河口区 ${ }^{226} \mathrm{Ra}$ 解吸活度最大值发生在盐度为 $9 \%$ 时, 长江口解吸活度最大值发生在 盐度为 $9 \%$ 时, 密西西比河解吸活度最大值发生在盐度为 $5 \%$ 时 ${ }^{[43-44]}$, Cable 等研究了海底沉积物 Ra 的解 吸, 认为在盐度为 $18 \%$ 左右 $R a$ 的解吸最强烈 ${ }^{[25]}$. 但是都有一个相同的规律, 就是随着盐度的升高, 镭同位 素的解吸量先增大, 达到一个最大值后开始减小.

Kiro 等对死海沿岸含水层 Ra 的分布模式研究发现, 短半衰期 Ra 同位素的活度随着盐度的降低而降 
表 1 布哈河河口悬浮颗粒物 Ra 的解吸量

Tab.1 Desorption Radium activity of suspended particles in the Buha River estuary

\begin{tabular}{lcccccc}
\hline & 实际盐度/\% & $\mathrm{pH}$ 值 & ${ }^{224} \mathrm{Ra} /(\mathrm{dpm} / \mathrm{g})$ & ${ }^{223} \mathrm{Ra} /(\mathrm{dpm} / \mathrm{g})$ & ${ }^{226} \mathrm{Ra} /(\mathrm{dpm} / \mathrm{g})$ & ${ }^{228} \mathrm{Ra} /(\mathrm{dpm} / \mathrm{g})$ \\
\hline $\mathrm{S}-0.0$ & 0.1 & - & $0.43 \pm 0.02$ & $0.018 \pm 0.001$ & $0.29 \pm 0.014$ & $0.14 \pm 0.07$ \\
$\mathrm{~S}-2.8$ & 3.1 & 8.27 & - & - & $0.15 \pm 0.004$ & $0.11 \pm 0.02$ \\
$\mathrm{~S}-5.8$ & 5.7 & 8.92 & $0.57 \pm 0.03$ & - & $0.18 \pm 0.004$ & $0.14 \pm 0.02$ \\
$\mathrm{~S}-8.8$ & 8.9 & 9.01 & $0.59 \pm 0.03$ & $0.025 \pm 0.001$ & $0.16 \pm 0.008$ & $0.18 \pm 0.03$ \\
$\mathrm{~S}-11.8$ & 12.0 & 9.03 & $0.37 \pm 0.02$ & $0.069 \pm 0.003$ & $0.21 \pm 0.006$ & $0.23 \pm 0.03$ \\
$\mathrm{~S}-14.8$ & 14.5 & 8.83 & $0.32 \pm 0.02$ & $0.036 \pm 0.002$ & $0.13 \pm 0.017$ & $0.17 \pm 0.03$ \\
八门湾 & - & - & - & - & 3.93 & - \\
胶州湾 & - & - & $0.32 \sim 0.98$ & - & $0.07 \sim 0.20$ & - \\
九江河口 & - & - & $0.11 \sim 0.61$ & - & $0.13 \sim 0.25$ & - \\
\hline
\end{tabular}

低,是由于盐度对 Ra 同位素解吸有影响 ${ }^{[45]}$. 辽河口水体中的 ${ }^{224} \mathrm{Ra}$ 和 ${ }^{223} \mathrm{Ra}$ 随盐度变化呈不保守现象, 盐度 为 $10 \%$ 左右的海区 Ra 同位素高峰值可能是悬浮颗粒物解吸造成的 ${ }^{[36]}$. Moore 对 Chesapeake 湾的 Ra 同位 素研究时, 发现该湾内水体中高 ${ }^{226} \mathrm{Ra}$ 和 ${ }^{228} \mathrm{Ra}$ 活度值主要来源于海底表层沉积物的扩散和河口颗粒物的解 吸 ${ }^{[46]}$. 对 Amazon 河口 ${ }^{226} \mathrm{Ra}$ 和 ${ }^{228} \mathrm{Ra}$ 行为的研究发现, 来自河口及附近陆架沉积物的 Ra 扩散提供了 Amazon 体系总 $\mathrm{Ra}$ 的 $1 / 3$, 其余 $2 / 3$ 来自河水溶解态及河流悬浮颗粒的解吸 ${ }^{[47]}$. Astwood 通过一系列解吸实验得出 亚马逊河口区有 $40 \%$ 的 ${ }^{224} \mathrm{Ra}$ 来自悬浮颗粒的解吸 ${ }^{[48]}$. Krest 等对 Mississippi 河和 Atchafalaya 河与海水混合 过程进行研究, 发现悬浮颗粒物上有大约 $50 \%$ 的 ${ }^{226} \mathrm{Ra}$ 和 ${ }^{228} \mathrm{Ra}$ 发生解吸, 其解吸活度达到 $18.0 \mathrm{dpm} / 100$ $\mathrm{L}^{[22]}$. 苏妮等研究海南东部鹓湖以及河口海底地下水发现, 万泉河口区 ${ }^{226} \mathrm{Ra}$ 的最大解活度为 $3.81 \mathrm{dpm} / 100$ $\mathrm{L}$, 八门湾地区 ${ }^{226} \mathrm{Ra}$ 的最大解吸活度为 $3.42 \mathrm{dpm} / 100 \mathrm{~L}$, 解吸态的 Ra 的活度不可忽 略 $^{[16,49]}$. 布哈河悬浮颗粒 物 ${ }^{226} \mathrm{Ra}$ 的最大解吸量达 $0.21 \mathrm{dpm} / \mathrm{g}$, 而河流平均含沙量为 $0.41 \mathrm{~g} / \mathrm{L}$, 那么河流悬浮颗粒物中 ${ }^{226} \mathrm{Ra}$ 的最大解 吸活度为 $8.4 \mathrm{dpm} / 100 \mathrm{~L}$, 布哈河水体中溶解态 ${ }^{226} \mathrm{Ra}$ 的活度为 $8.30 \pm 0.24 \sim 21.63 \pm 0.37 \mathrm{dpm} / 100 \mathrm{~L}$, 河流悬浮 颗粒物中 ${ }^{226} \mathrm{Ra}$ 的解吸活度占到了河流水体中 ${ }^{226} \mathrm{Ra}$ 活度的 $38.83 \%$. 由此可见, 布哈河河口悬浮颗粒物解吸 的 Ra 是青海湖水体中 $R a$ 的一项不可忽略的来源, 而且在青海湖表层水体中 Ra 同位素的活度分布呈现出 由沿岸向湖中心跳跃式的减少 ${ }^{[50]}$, 也可能是受到颗粒物解吸的影响.

从布哈河河口悬浮颗粒物中 $\mathrm{Ra}$ 同位素解吸量与 $\mathrm{pH}$ 值的关系可以看到, 随着 $\mathrm{pH}$ 值的增加, ${ }^{226} \mathrm{Ra}$ 和 ${ }^{228} \mathrm{Ra}$ 的解吸量呈逐渐增高趋势, 趋势与盐度对其解吸影响一致 (图 3b). 实验结果显示, $\mathrm{pH}$ 值对悬浮颗粒物 中 ${ }^{224} \mathrm{Ra} 、{ }^{223} \mathrm{Ra} 、{ }^{226} \mathrm{Ra} 、{ }^{228} \mathrm{Ra}$ 解吸的影响不同. 在 $\mathrm{pH}$ 值小于 8.8 的情况下没检测到 ${ }^{223} \mathrm{Ra}$ 和 ${ }^{224} \mathrm{Ra}$ 的解吸量, 对 于 ${ }^{223} \mathrm{Ra} 、{ }^{224} \mathrm{Ra}$ 的解吸量与 $\mathrm{pH}$ 值的关系不好解释. ${ }^{226} \mathrm{Ra}$ 和 ${ }^{228} \mathrm{Ra}$ 解吸量随着 $\mathrm{pH}$ 值的升高表现出明显的增加 趋势. Beck 等在美国弗吉尼亚州 Gloucester Point 沙滩实验的研究表明, 孔隙水的 $\mathrm{pH}$ 值对 Ra 解吸的影响可 以与盐度对 $\mathrm{Ra}$ 解吸的影响相提并论, 当 $\mathrm{pH}$ 值在 $2 \sim 10$ 范围内时, $\mathrm{Ra}$ 的固液分配系数可以变化 $1 \sim 2$ 个数量 级, 当 $\mathrm{pH}$ 值的突跃范围为 $5 \sim 8$ 时, $\mathrm{pH}$ 值微小变动将引起 $\mathrm{Ra}$ 的固液分配系数发生 10 倍左右的变化 ${ }^{[27]}$. 而 在青海湖研究区, 布哈河河水的 $\mathrm{pH}$ 值变化范围为 8.19 8.59, 青海湖湖水 $\mathrm{pH}$ 值变化范围为 $9.02 \sim 9.30$, 从河 水到湖水 $\mathrm{pH}$ 值较小的范围内 Ra 的解吸量与盐度为 $2 \% 0 \sim 14.8 \% 0$ 时 Ra 的解吸量一样. 因此, 从本实验也可 以看出, $\mathrm{pH}$ 值的变化对青海湖 Ra 解吸的影响较大,并且比盐度对 Ra 解吸的影响更明显 (图 4).

\section{2 河流底部沉积物和湖底沉积物中镭同位素的扩散特征}

不同培养时间段的河流和湖底沉积物 Ra 扩散量见表 2 , 由于本次实验检测的 ${ }^{224} \mathrm{Ra}$ 活度是总的 ${ }^{224} \mathrm{Ra}$, 未 检测并去掉母体中 ${ }^{224} \mathrm{Ra}$ 的活度值, 计算出的 ${ }^{224} \mathrm{Ra}$ 活度值不准确, 在此不再对 ${ }^{224} \mathrm{Ra}$ 的扩散速率进行考虑. 从 培养时间与扩散通量的关系可以看出, 扩散开始时河流中 ${ }^{226} \mathrm{Ra}$ 和 ${ }^{228} \mathrm{Ra}$ 的活度比较高, 随着扩散时间的增加 表现出降低的趋势, 扩散 $96 \mathrm{~h}$ 后, 又呈现增加的趋势, 最后趋于稳定(图 5). 这种现象可能是由于 Ra 同位素 的解吸反应在几秒到几小时内就可以完成, 当培养时间较短时, Ra 的活度普遍偏高, 且没有规律可循, 因此, 培养刚开始时镭的活度主要来自沉积物中原有镭同位素的解吸,来自扩散的 Ra 不明显. 过一段时间后, 沉 积物间隙水中 $\mathrm{Ra}$ 活度与新加人的无 Ra 河口水 Ra 活度之差达到最大, Ra 活度梯度也达到最大, 这就会促 

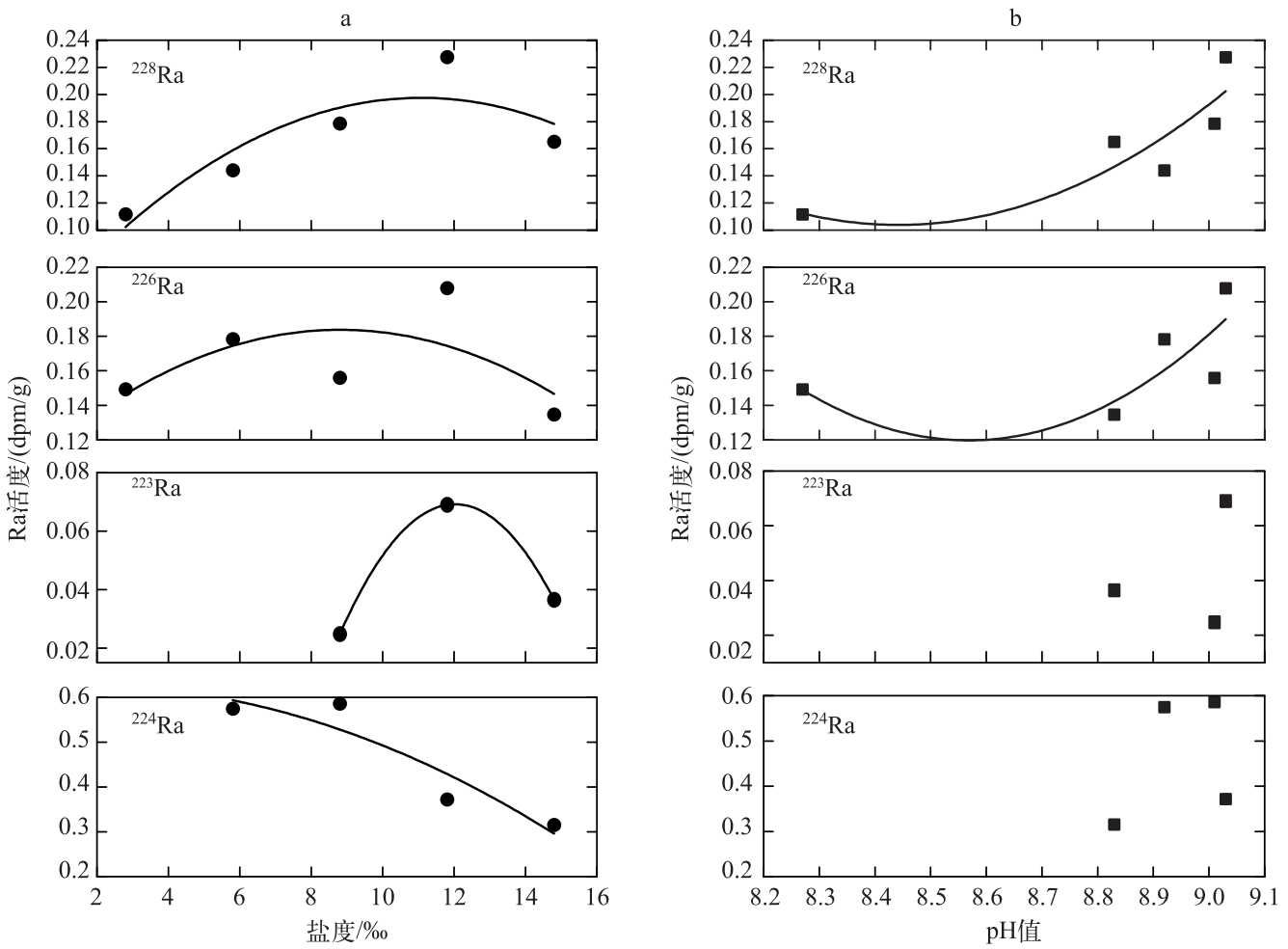

图 3 布哈河河口悬浮颗粒物中镭解吸量与盐度 (a) 和 $\mathrm{pH}$ 值 (b) 的关系

Fig.3 Relationships between desorption Radium activity of suspended particles and salinity (a) and $\mathrm{pH}$ values (b) in Buha River estuary

使分子扩散作用加剧, 沉积物孔隙水中 Ra 同位素的扩 散作用开始增强, 孔隙水中 Ra 同位素浓度开始增 $大^{[26]}$. 即湖底和河流沉积物样品中的 Ra 同位素通过 其母体 (Th) 的自然衰变而生成, 通过解吸作用进人到 间隙水中, 然后在密度差的作用下向上覆水体扩 散 ${ }^{[29]}$, 在水体中垂直分布的表现为, Ra 活度表层高, 随 深度增大而逐渐降低, 达到一定深度, 随深度的增大而 增高 ${ }^{[51]}$. 由于其半衰期很长, 致使上覆水体中 ${ }^{226} \mathrm{Ra}$ 的 添加十分缓慢, 所以短时间内不会出现扩散输人与衰 变之间的平衡, 在图上的反映就是散点基本上呈现缓 慢增加的趋势.

我们假设上覆水体中只存在沉积物扩散和放射衰 变,并且沉积物释放出的 $\mathrm{Ra}$ 大于放射性衰变损失的 量, 也就是说在沉积物的上覆水体中 $\mathrm{Ra}$ 活度变化的结 果是一个正值,那么这个值可以表示为:

$$
\mathrm{d} I / \mathrm{d} t=J_{\text {diff }} \cdot A_{\text {surf }}-\lambda \cdot I
$$

通过积分可以得到:

$$
I=\left(J_{\text {diff }} \cdot A-\mathrm{e}^{-\lambda \cdot t}\right) / \lambda
$$

而上覆水体中 $\mathrm{Ra}$ 同位素的活度随时间的变化可以表达为 ${ }^{[52]}$ :

$$
I_{t}=\left(J_{\text {diff }} \cdot A_{\text {diff }} / \lambda\right)\left(1-\mathrm{e}^{-\lambda \cdot t}\right)+I_{0} \cdot \mathrm{e}^{-\lambda \cdot t}
$$


式中, $I_{0}$ 和 $I_{t}$ 是初始时刻和 $t$ 时刻上覆水体中 $R \mathrm{R}$ 的库存量 $(\mathrm{dpm}), J_{\mathrm{diff}}$ 是单位面积、单位时间内 Ra 的扩散通 量 $\left(\mathrm{dpm} /\left(\mathrm{m}^{2} \cdot \mathrm{h}\right)\right), \lambda$ 是镭的衰变常数 $\left(\mathrm{h}^{-1}\right), A_{\mathrm{diff}}$ 是培养样品的面积 ( 河流 $=0.1 \mathrm{~m}^{2}$, 湖底沉积物 $\left.=0.05 \mathrm{~m}^{2}\right)$. 对于长半衰期 ${ }^{226} \mathrm{Ra}$ 和 ${ }^{228} \mathrm{Ra}$ 来说, 由于其半衰期很长, 在培养时间范围的由放射衰变损失的量可以忽略. 因 此 ${ }^{226} \mathrm{Ra}$ 和 ${ }^{228} \mathrm{Ra}$ 的活度随时间的变化公式为:

$$
I_{t}=J_{\text {diff }} \cdot A_{\text {surf }} \cdot t
$$

根据理论公式 (8) 和 (9) 用拟合法来确定布哈河沉积物和青海湖底部沉积物培养样的 $J_{\text {diff }}$ 值, 得到布哈 河和青海湖湖底沉积物样品的 Ra 扩散速率(表 2). 河流沉积物 ${ }^{226} \mathrm{Ra}$ 和 ${ }^{228} \mathrm{Ra}$ 扩散速率分别是 0.039 和 0.29 $\mathrm{dpm} /\left(\mathrm{m}^{2} \cdot \mathrm{h}\right)$; 湖底沉积物 ${ }^{226} \mathrm{Ra}$ 和 ${ }^{228} \mathrm{Ra}$ 扩散速率分别是 0.018 和 $0.092 \mathrm{dpm} /\left(\mathrm{m}^{2} \cdot \mathrm{h}\right)$. 河流底部沉积物中 $R a$ 的扩散速率要比湖底沉积物中 $R a$ 的扩散速率快, 可能是因为湖泊底部表层沉积物长时间在咸水中, Ra 同位素早已经发生解吸, 沉积物中 ${ }^{226} \mathrm{Ra}$ 的扩散速率比 ${ }^{228} \mathrm{Ra}$ 小, 是由于 ${ }^{226} \mathrm{Ra}$ 的再生速率很慢, 上覆水体中 ${ }^{226} \mathrm{Ra}$ 的添加十分缓慢造成的.

表 2 布哈河河口和青海湖湖底沉积物中 Ra 同位素扩散通量

Tab.2 Diffusion flux of Radium from sediments of Buha River estuary and Lake Qinghai

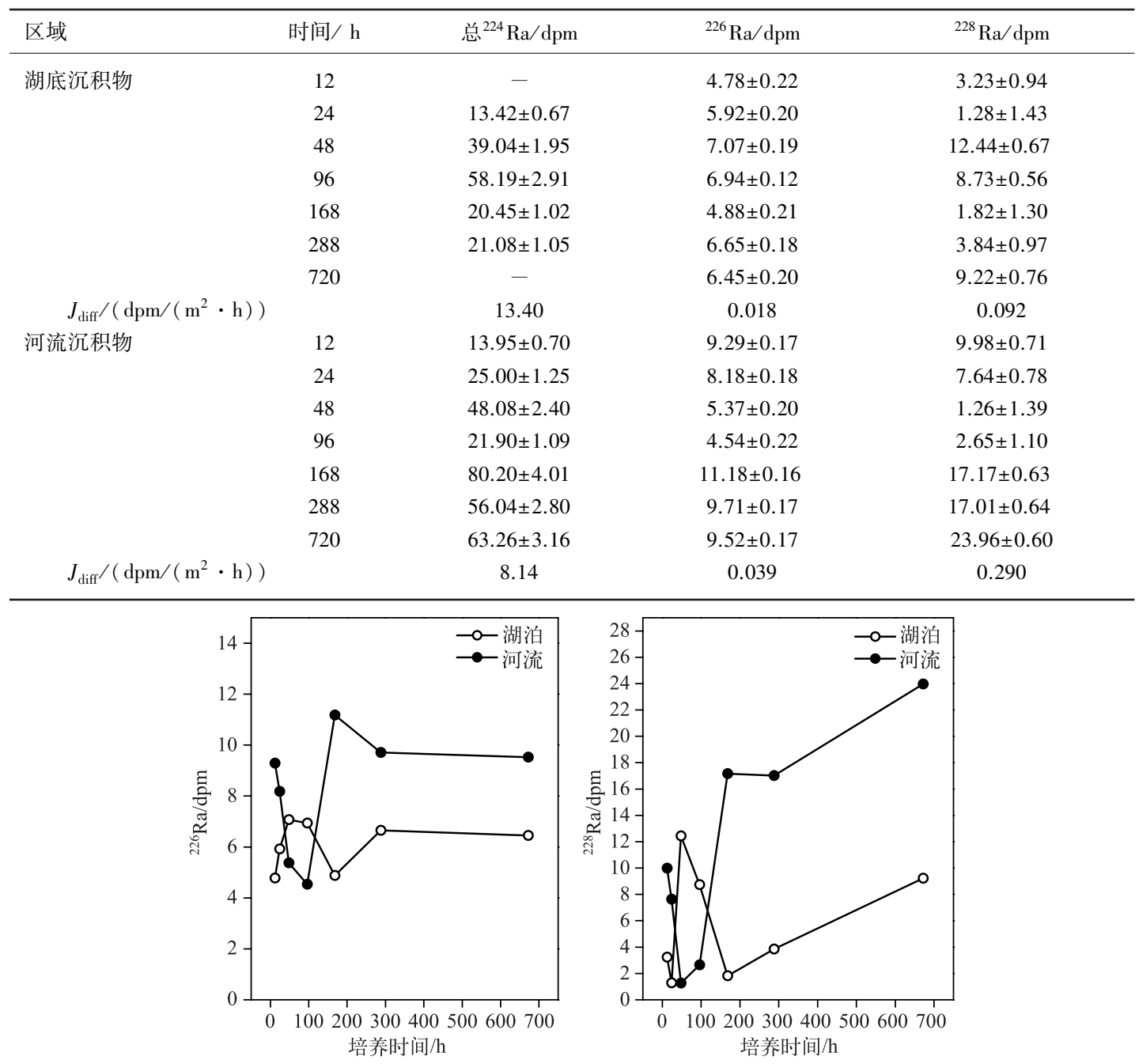

图 5 布哈河河口沉积物中 Ra 扩散量与培养时间的关系

Fig.5 Relationship between Radium diffusion flux in sediments of Buha River estuary and time 
Garcia-Solsona 等用意大利 Venice 瀉湖北部盐沼的沉积物做了扩散实验,得到沉积物中 ${ }^{226} \mathrm{Ra}$ 和 ${ }^{228} \mathrm{Ra}$ 的 扩散通量分别为 0.28 和 $0.45 \mathrm{~Bq} /\left(\mathrm{m}^{2} \cdot \mathrm{d}\right)^{[51]}$. 郭占荣等对九龙江河口沉积物进行了扩散实验, 得到九龙江 河口沉积物中 ${ }^{226} \mathrm{Ra}$ 的扩散通量为 $0.04 \mathrm{~Bq} /\left(\mathrm{m}^{2} \cdot \mathrm{d}\right)^{[52]}$. Beck 等对 Jamaica 湾潮间带的沉积物镭扩散实验结 果显示沉积物中 ${ }^{223} \mathrm{Ra}$ 和 ${ }^{224} \mathrm{Ra}$ 的扩散通量分别为 0.02 和 $0.47 \mathrm{~Bq} /\left(\mathrm{m}^{2} \cdot \mathrm{d}\right)$, Jamaica 湾水体中的 Ra 有 $4 \% \sim$ $11 \%$ 来源于海底沉积物的扩散输人 ${ }^{[40]}$. 布哈河河口和青海湖湖底沉积物的 ${ }^{226} \mathrm{Ra}$ 和 ${ }^{228} \mathrm{Ra}$ 扩散速率与沿海地 区相比较小, 比胶州湾的略小,但是在同一个数量级上(表 3).

表 3 青海湖沉积物中 Ra 的扩散速率与其他地区的对比

Tab.3 Diffusion rate of Radium in sediments of Lake Qinghai compared with that of other areas

\begin{tabular}{lcccc}
\hline \multirow{2}{*}{ 地区 } & 沉积物类型 & \multicolumn{3}{c}{$J_{\text {diff }} /\left(\mathrm{dpm} /\left(\mathrm{m}^{2} \cdot \mathrm{h}\right)\right)$} \\
\cline { 3 - 5 } & & ${ }^{224} \mathrm{Ra}$ & ${ }^{226} \mathrm{Ra}$ & 0.290 \\
\hline 布哈河河口 & 河流 & - & 0.039 & 0.092 \\
青海湖底部 $^{\text {九龙江河口 }}{ }^{[53]}$ & 湖底 & - & 0.018 & - \\
VeniceLagoon $^{[52]}$ & 河流 & 0.950 & 0.110 & 1.130 \\
胶州湾 $^{[29]}$ & 盐沼 & 1.330 & 0.710 & - \\
\hline
\end{tabular}

沉积物扩散出的镭同位素通量可以用下式进行计算:

$$
I_{\text {diff }}=J_{\text {diff }} \cdot A_{\text {sed }} \cdot H_{\text {day }}
$$

式中, $J_{\text {diff }}$ 是单位面积、单位时间内沉积物扩散的镭通量 $\left(\mathrm{dpm} /\left(\mathrm{m}^{2} \cdot \mathrm{h}\right)\right), A_{\mathrm{sed}}$ 是表层沉积物的覆盖面积 $\left(\mathrm{m}^{2}\right), H_{\mathrm{day}}$ 是每天的小时数 $(24 \mathrm{~h})$.

研究区域青海湖南湾和北湾的水体覆盖面积分别为 $4.30 \times 10^{8}$ 和 $1.51 \times 10^{8} \mathrm{~m}^{2}$. 根据公式 (10) 可以算出 青海湖北湾湖底沉积物每天释放出的 ${ }^{226} \mathrm{Ra}$ 和 ${ }^{228} \mathrm{Ra}$ 通量分别为 $1.86 \times 10^{8}$ 和 $9.49 \times 10^{8} \mathrm{dpm}$, 南湾分别为 $6.52 \times$ $10^{7}$ 和 $3.33 \times 10^{8} \mathrm{dpm}$, 如果换成浓度的话, 北湾和南湾通过湖底沉积物扩散的 ${ }^{226} \mathrm{Ra}$ 和 ${ }^{228} \mathrm{Ra}$ 的活度分别是 0.005 和 $0.025 \mathrm{dpm} / 100 \mathrm{~L}$, 布哈河集流面积为 $1.43 \times 10^{10} \mathrm{~m}^{2}$, 同样根据公式 (3) (10) 可以算出布哈河河底沉积物 每天释放出的 ${ }^{226} \mathrm{Ra}$ 和 ${ }^{228} \mathrm{Ra}$ 通量分别为 $1.34 \times 10^{10}$ 和 $9.95 \times 10^{10} \mathrm{dpm}$, 换成浓度的话, 通过河底沉积物扩散 ${ }^{226} \mathrm{Ra}$ 和 ${ }^{228} \mathrm{Ra}$ 的活度分别是 1.7 和 $12.6 \mathrm{dpm} / 100 \mathrm{~L}$, 分别占到河流溶解态 ${ }^{226} \mathrm{Ra}$ 和 ${ }^{228} \mathrm{Ra}$ 的 $8.0 \%$ 和 $37.80 \%$. 由 此可见, 青海湖湖底沉积物中 Ra 同位素扩散的活度与河流和地下水体中溶解态的 Ra 活度相比非常小, 比 误差值还小, 所以湖底沉积物扩散的 Ra 活度几乎可以忽略, 而布哈河河底沉积物扩散的 Ra 活度是不可以 忽略的.

\section{4 结论}

1) 通过对布哈河河口悬浮颗粒物中 Ra 进行解吸实验, 得到了不同盐度 $(2.8 \%$ 、 $5.8 \%$ 、 $8.8 \%$ 、 $11.8 \%$ 和 $14.8 \%$ ) 布哈河悬浮颗粒物中 $\mathrm{Ra}$ 的解吸活度, 其中 ${ }^{223} \mathrm{Ra} 、{ }^{224} \mathrm{Ra} 、{ }^{226} \mathrm{Ra}$ 和 ${ }^{228} \mathrm{Ra}$ 的解吸活度分别为 $0.018 \pm 0.001 \sim$ $0.069 \pm 0.003 、 0.32 \pm 0.02 \sim 0.59 \pm 0.03 、 0.13 \pm 0.017 \sim 0.29 \pm 0.014$ 和 $0.11 \pm 0.02 \sim 0.23 \pm 0.03 \mathrm{dpm} / \mathrm{g} .{ }^{224} \mathrm{Ra}$ 的解吸 活度均高于 ${ }^{226} \mathrm{Ra}$ 和 ${ }^{228} \mathrm{Ra}$ 的解吸活度.

2) 在固一液分配体系中随着盐度的逐渐增加, 解吸态 Ra 同位素活度呈增加趋势, 在盐度为 $12 \%$ 附近时 布哈河悬浮颗粒物中 ${ }^{226} \mathrm{Ra}$ 和 ${ }^{228} \mathrm{Ra}$ 的解吸活度达到了最大值, 当盐度 $<9 \%$ 时, ${ }^{226} \mathrm{Ra}$ 的解吸活度大于 ${ }^{228} \mathrm{Ra}$, 当 盐度 $>9 \%$ 时, ${ }^{228} \mathrm{Ra}$ 的解吸活度大于 ${ }^{226} \mathrm{Ra}$, 这可能与当地富铀矿有关. 当盐度 $<4 \%$ 和盐度 $>12 \%$ 时, $\mathrm{Ra}$ 的解吸 量增加较为缓慢, 当 $4 \% 0<$ 盐度 $<12 \%$ 时, $R a$ 的解吸量增加较迅速. $\mathrm{pH}$ 值的变化对青海湖 Ra 解吸的影响较 大, 并且比盐度对 Ra 解吸的影响更明显.

3) 通过扩散实验,得到了布哈河和青海湖湖底沉积物中 ${ }^{226} \mathrm{Ra}$ 和 ${ }^{228} \mathrm{Ra}$ 的扩散速率. 河流沉积物中 ${ }^{226} \mathrm{Ra}$ 和 ${ }^{228} \mathrm{Ra}$ 的扩散速率分别是 0.039 和 $0.290 \mathrm{dpm} /\left(\mathrm{m}^{2} \cdot \mathrm{h}\right)$; 湖底沉积物 ${ }^{226} \mathrm{Ra}$ 和 ${ }^{228} \mathrm{Ra}$ 的扩散速率分别是 0.018 和 $0.092 \mathrm{dpm} /\left(\mathrm{m}^{2} \cdot \mathrm{h}\right)$. 湖底沉积物扩散活度小于河流沉积物扩散活度, 在分析青海湖水体中 Ra 同位素的 来源时,湖底沉积物扩散量可以忽略,而河流底部沉积物中 Ra 同位素的扩散量是不可以忽略的. 


\section{5 参考文献}

[ 1 ] Rama, Moore WS. Mechanism of transport of U-Th series radioisotopes from solids into ground water. Geochimica et Cosmochimica Acta, 1984, 48(2) : 395-399.

[ 2 ] Porcelli D, Swarzenski PW. The behavior of U-and Th-series nuclides in groundwater. Reviews in Mineralogy and Geochemistry, 2003, 52(1): 317-361.

[ 3 ] Suksi J, Rasilainen K, Casanova J et al. U-series disequilibria in a groundwater flow route as an indicator of uranium migration processes. Journal of Contaminant Hydrology, 2001, 47(2) : 187-196.

[ 4 ] Zhang Lei. Radium isotopes in Changjiang Estuary/East China Sea and their applieationin analysis of ingamong multiple water masses[Dissertation]. Hangzhou: Zhejiang University, 2007 (in Chinese with English abstract). [张砟. 长江口、东 海的镭同位素及其在水团混合分析中的应用 [学位论文]. 杭州: 浙江大学, 2007.]

[ 5 ] Dulaiova H, Burnett WC. Evaluation of the flushing rates of Apalachicola Bay, Florida via natural geochemical tracers. Marine Chemistry, 2008, 109(3) : 395-408.

[ 6 ] Moore WS. Determining coastal mixing rates using radium isotopes. Continental Shelf Research, 2000, 20(15) : 1993-2007.

[ 7 ] Moore WS. Fifteen years experience in measuring ${ }^{224} \mathrm{Ra}$ and ${ }^{223} \mathrm{Ra}$ by delayed-coincidence counting. Marine Chemistry, $2008, \mathbf{1 0 9}(3)$ : 188-197.

[ 8 ] Peterson RN, Burnett WC, Taniguchi M et al. Determination of transport rates in the Yellow River-Bohai Sea mixing zone via natural geochemical tracers. Continental Shelf Research, 2008, 28(19) : 2700-2707.

[ 9 ] Burnett W, Aggarwal P, Aureli A et al. Quantifying submarine groundwater discharge in the coastal zone via multiple methods. Science of the Total Environment, 2006, 367(2) : 498-543.

[10] Burnett WC, Peterson R, Moore WS et al. Radon and radium isotopes as tracers of submarine groundwater discharge-results from the Ubatuba, Brazil SGD assessment intercomparison. Estuarine, Coastal and Shelf Science, 2008, 76( 3 ): 501-511.

[11] Moore WS. Large groundwater inputs to coastal waters revealed by ${ }^{226}$ Ra enrichments. Nature, 1996, 380 ( 6575$)$ : 612-614.

[12] Rama, Moore WS. Using the radium quartet for evaluating groundwater input and water exchange in salt marshes. Geochimica et Cosmochimica Acta, 1996, 60(23) : 4645-4652.

[13] Moore WS. Sources and fluxes of submarine groundwater discharge delineated by radium isotopes. Biogeochemistry, 2003, 66 $(1 / 2): 75-93$.

[14] Swarzenski PW, Reich C, Kroeger KD et al. Ra and Rn isotopes as natural tracers of submarine groundwater discharge in Tampa Bay, Florida. Marine Chemistry, 2007, 104(1/2) : 69-84.

[ 15] Su N, Du J, Li Y et al. Evaluation of surface water mixing and associated nutrient fluxes in the East China Sea using ${ }^{226} \mathrm{Ra}$ and ${ }^{228}$ Ra. Marine Chemistry, 2013, 156: 1-12.

[16] Su N, Du JZ, Moore WS et al. An examination of groundwater discharge and the associated nutrient fluxes into the estuaries of eastern Hainan Island, China using ${ }^{226}$ Ra. Science of the Total Environment, 2011, 409(19) : 3909-3918.

[17] Krest JM, Harvey JW. Using natural distributions of short-lived radium isotopes to quantify groundwater discharge and recharge. Limnology and Oceanography, 2003, 48(1) : 290-298.

[18] Webster IT, Hancock GJ, Murray AS. Modelling the effect of salinity on radium desorption from sediments. Geochimica et Cosmochimica Acta, 1995, 59(12) : 2469-2476.

[19] Dukat DA, Kuehl SA. Non-steady-state ${ }^{210} \mathrm{~Pb}$ flux and the use of ${ }^{228} \mathrm{Ra},{ }^{226} \mathrm{Ra}$ as a geochronometer on the Amazon continental shelf. Marine Geology, 1995, 125(3) : 329-350.

[20] Su CC, Huh CA. ${ }^{210} \mathrm{~Pb},{ }^{137} \mathrm{Cs}$ and ${ }^{239,240} \mathrm{Pu}$ in East China Sea sediments: sources, pathways and budgets of sediments and radionuclides. Marine Geology, 2002, 183(1) : 163-178.

[21] Watters DL, Kline DE, Coale KH et al. Radiometric age confirmation and growth of a deep-water marine fish species: The bank rockfish, Sebastes rufus. Fisheries Research, 2006, 81(2) : 251-257.

[22] Krest JM, Moore WS. ${ }^{226}$ Ra and ${ }^{228}$ Ra in the mixing zones of the Mississippi and Atchafalaya Rivers: indicators of groundwater input. Marine Chemistry, 1999, 64(3): 129-152. 
[23] Li YH, Mathieu G, Biscaye P et al. The flux of ${ }^{226}$ Ra from estuarine and continental shelf sediments. Earth and Planetary Science Letters, 1977, 37(2) : 237-241.

[24] Langmuir D, Riese AC. The thermodynamic properties of radium. Geochimica et Cosmochimica Acta, 1985, 49(7): 1593-1601.

[25] Cable JE, Smith CG, Blanford WJ. Measurements of dispersivity and retardation factors in marine sediments using tritiated calcium chloride solution and Radium-226. Radioprotection, 2009. 44(5) : 185-190.

[26] Gonneea ME, Morris PJ, Dulaiova H et al. New perspectives on radium behavior within a subterranean estuary. Marine Chemistry, 2008, 109(3) : 250-267.

[27] Beck AJ, Cochran MA. Controls on solid-solution partitioning of radium in saturated marine sands. Marine Chemistry, 2013, 156: 38-48.

[28] Smith B, Amonette A. The environmental transport of radium and plutonium: a review. Maryland: Institute for Energy and Environmental Research, 2006.

[29] Yuan Xiaojie, Guo Zhanrong, Liu Jie et al. Characteristics of radium desorption from sediments in the salt water environment. Acta Geoscientica Sinica, 2014, 35(5) : 582-588 (in Chinese with English abstract). [袁晓婕, 郭占荣, 刘洁等. 咸水环境下沉积物中镭的解吸特点. 地球学报, 2014, 35(5): 582-588.]

[30] Wang R, Chau A, Liu F et al. Studies on the adsorption and migration of radium in natural minerals. Journal of Radioanalytical and Nuclear Chemistry, 1993, 171(2): 347-364.

[31] Sun Y, Torgersen T. Adsorption-desorption reactions and bioturbation transport of ${ }^{224}$ Ra in marine sediments : a one-dimensional model with applications. Marine chemistry, 2001, 74(4) : 227-243.

[32] Turekian KK. Radium on soil mineral surfaces: Its mobility under environmental conditions and its role in radon emanation. Final report. Yale Univ. Funding organisation: USDOE Office of Energy Research, Washington, DC ( United States) : New Haven, CT (United States), 1997.

[33 ] Krupka K, Serne R. Understanding variation in partition coefficient, Kd, values, Volume III : Review of geochemistry and available Kd values for americium, arsenic, curium, iodine, neptunium, radium, and technetium. Richland: Pacific Northwest National Laboratory, 2000, 285: 271-277.

[34] Vinson DS. Vengosh A, Hirschfeld D et al. Relationships between radium and radon occurrence and hydrochemistry in fresh groundwater from fractured crystalline rocks, North Carolina (USA). Chemical Geology, 2009, 260(3) : 159-171.

[ 35 ] Tomita J, Zhang J, Yamamoto M. Radium isotopes ( ${ }^{226} \mathrm{Ra}$ and $\left.{ }^{228} \mathrm{Ra}\right)$ in Na-Cl type groundwaters from Tohoku District (Aomori, Akita and Yamagata Prefectures) in Japan. Journal Of Environmental Radioactivity, 2014, 137: 204-212.

[36] Xu Bochao. Preconcentration and determination of radium isotopes and their applications as tracers to assess water mixing processes in estuaries[Dissertation]. Qingdao: Ocean University of China, 2011 (in Chinese with English abstract). [许博 超, 天然镭同位素富集和测定方法及对河口混合过程的示踪研究 [学位论文]. 青岛: 中国海洋大学, 2011.]

[37] Martin P, Akber RA. Radium isotopes as indicators of adsorption-desorption interactions and barite formation in groundwater. Journal of Environmental Radioactivity, 1999, 46(3) : 271-286.

[ 38 ] Tsezos M, Keller D. Adsorption of radium 226 by biological origin absorbents. Biotechnology and Bioengineering, 1983,25 (1) : 201-215.

[39] Huang Lei. Researehon groundwater discharge into Jiulongjiang Estuary [Dissertation]. Xiamen: Xiamen University, 2009 (in Chinese with English abstract).[黄磊. 九龙江河口区的地下水输人研究 [学位论文]. 厦门: 厦门大学, 2009.]

[40] Beck AJ, Tsukamoto Y, Tovar-Sanchez A et al. Importance of geochemical transformations in determining submarine groundwater discharge-derived trace metal and nutrient fluxes. Applied Geochemistry, 2007, 22(2) : 477-490.

[41] Lanzhou Branch Chinese Acdemy of Scienceseienee ed. Evolution of recent environment in Qinghai lake and its prediction. Beijing: Science Press, 1994: 270(in Chinese). [中国科学院兰州分院. 青海湖近代环境的演化和预测. 北京: 科学 出版社, 1994: 270.]

[42] Su Ni. Tracing coastal water mixing processes and submarine groundwater discharge by radium isotopes [Dissertation]. Shanghai : East China Normal University, 2013(in Chinese with English abstract). [苏妮. 镭同位素示踪的近岸水体混 合和海底地下水排泄 [学位论文]. 上海: 华东师范大学, 2013.]

[43] Elsinger RJ, Moore WS. ${ }^{226} \mathrm{Ra}$ and ${ }^{228} \mathrm{Ra}$ in the mixing zones of the Pee Dee River-Winyah bay, Yangtze River and Delaware bay estuaries. Estuarine, Coastal and Shelf Science, 1984, 18(6) : 601-613. 
[44] Shi Wenyuan, Qiu Xiaohui, Huang Yipu. The distribution of soluble ${ }^{226}$ Ra in Jiulongjiang-Xiamen Bay. Acta Oceanologica Sinica , 1993, 15(4) : 50-55(in Chinese with English abstract). [施文远, 邱晓晖, 黄奕普. 九龙江一厦门湾河口区溶 解 ${ }^{226} \mathrm{Ra}$ 的分布. 海洋学报, $1993, \mathbf{1 5}(4): 50-55$. ]

[45] Kiro Y, Yechieli Y, Voss CI et al. Modeling radium distribution in coastal aquifers during sea level changes: The Dead Sea case. Geochimica et Cosmochimica Acta, 2012, 88: 237-254.

[46] Moore WS. Radium isotopes in the Chesapeake Bay. Estuarine, Coastal and Shelf Science, 1981, 12(6) : 713-723.

[47] Key R, Stallard R, Moore W et al. Distribution and flux of ${ }^{226} \mathrm{Ra}$ and ${ }^{228} \mathrm{Ra}$ in the Amazon River estuary. Journal of Geophysical Research, 1985, 90(4) : 6995-7004.

[48] Astwood HM. The desorption of radium from Amazon sediment [ Dissertation ]. Columbia: University of South Carolina, 1991.

[49] Kong FC. Analysis of the distribution characteristics of ${ }^{226} \mathrm{Ra}$ and ${ }^{228} \mathrm{Ra}$ and their sources in the western part of Qinghai Lake. Chinese Journal of Oceanology and Limnology, 2015, 33(6) : 1402-1412.

[50] Moore WS, Sarmiento JL, Key RM. Submarine groundwater discharge revealed by ${ }^{228}$ Ra distribution in the upper Atlantic Ocean. Nature Geoscience, 2008, 1(5): 309-311.

[51] Garcia-Solsona E, Masqué P, Garcia-Orellana J et al. Estimating submarine groundwater discharge around Isola La Cura, northern Venice Lagoon (Italy), by using the radium quartet. Marine Chemistry, 2008, 109(3) : 292-306.

[52] Guo Zhanrong, Huang Lei, Yaung Xiaojie et al. Estimating submarine groundwater discharge to the Jiulong River estuary using Ra isotopes. Advances in Water Science, 2011, 22(1) : 118-125. (in Chinese with English abstract). [ 郭占荣, 黄 否, 袁晓婕等. 用镭同位素评价九龙江河口区的地下水输人. 水科学进展, 2011, 22(1): 118-125.] 